\title{
TAPERED CAPILLARIES APPLIED IN LASER WAKEFIELD ACCELERATION*
}

S. Mark Wiggins, Salima Abuazoum, Gregory Vieux, Gregor H. Welsh, Riju C. Issac, M. Ranaul Islam, Bernhard Ersfeld, Enrico Brunetti, Silvia Cipiccia,

David W. Grant and Dino A. Jaroszynski

Scottish Universities Physics Alliance, Department of Physics, University of Strathclyde, Glasgow G4 ONG, United Kingdom

The gas-filled capillary discharge waveguide (CDW) is a commonly applied accelerating structure for the laser wakefield accelerator (LWFA) because an intense femtosecond laser pulse can be successfully guided over several centimetres, leading to the generation of GeV-scale electron beams. Simulations have shown that tapering the longitudinal plasma density leads to significant enhancement of the final electron energy in a single accelerating stage.

Here we present realisation of linearly tapered CDWs, manufactured using a femtosecond laser micromachining technique pioneered at the University of Strathclyde ${ }^{1}$. One demonstration alumina waveguide has a length of $40 \mathrm{~mm}$ and a diameter that decreases linearly from $320 \mu \mathrm{m}$ to $270 \mu \mathrm{m}$ along its length. During waveguide characterisation, plasma is generated upon application of a $20 \mathrm{kV}$ voltage pulse after hydrogen gas is injected into the capillary. The transverse plasma density profile at either end is obtained with a detection system measuring the Stark-broadened hydrogen spectral line emission. At a gas backing pressure of $60 \mathrm{mbar}$, the on-axis, time-integrated density increases from $0.8 \times$ $10^{18} \mathrm{~cm}^{-3}$ (wider end) to $1.5 \times 10^{18} \mathrm{~cm}^{-3}$ (narrower end) thus indicating a tapered longitudinal plasma density.

Waveguiding of a low power, $50 \mathrm{fs}$ duration laser pulse is demonstrated $^{2}$ and, despite a slight mismatch of the laser focal spot size with respect to the capillary entrance size, efficient guiding of the Gaussian-shaped laser pulse is obtained. Energy transmission of $80 \%$ is obtained for optimal delay of the laser pulse arrival time with respect to the discharge current pulse.

In high-power LWFA experiments, comparison is made, for identical laser conditions (intensity of $1.6 \times 10^{18} \mathrm{~W} / \mathrm{cm}^{2}$ ), between three tapering configurations: density decreasing with distance (negative taper), density constant (no taper) and density increasing with distance (positive taper), over the same capillary length of $40 \mathrm{~mm}$ in the (average) plasma density range of $3-6 \times 10^{18} \mathrm{~cm}^{-3}$.

1. S. M. Wiggins et al., Rev. Sci. Instrum. 82, 096104 (2011).

2. S. Abuazoum et al., Appl. Phys. Lett. 100, 014106 (2012).

* Work supported by U.K. EPSRC and STFC and EC Laserlab-Europe/Laptech and ELI projects. 